\title{
Temporal Arago spot in optical fibers
}

\author{
Christophe Finot ${ }^{* a}$ and Hervé Rigneault ${ }^{b}$ \\ ${ }^{a}$ Laboratoire Interdisciplinaire Carnot de Bourgogne, UMR 6303 CNRS - Université de Bourgogne- \\ Franche-Comté, 9 avenue Alain Savary, BP 47870, 21078 Dijon Cedex, France \\ b Aix Marseille Univ., CNRS, Centrale Marseille, Institut Fresnel UMR 7249, 13397 Marseille, \\ France
}

\begin{abstract}
The history of conceptions of light is among the most exciting scientific adventures. It was Fresnel's theoretical work that made it possible to establish the most solid theoretical basis of wave optics. Two centuries ago, his approach was validated by the observation of a counter-intuitive bright spot appearing at the center of the geometric shadow of an illuminated opaque circular object. This phenomenon has remained known as the Arago spot. We propose here to further extend the spatial/temporal analogy that exists between diffraction and dispersion by revisiting the Arago spot formation in the time domain through the temporal dispersive evolution of light after being briefly stopped by an obstacle. The analytical treatment that is possible for linear propagation as well as the experiments based on telecom optical fibers and fast optoelectronics confirm that we observe the emergence of light where it was initially absent. As the power increases and Kerr nonlinearity affects the propagation, the Arago spot intensity is affected by the sign of the dispersion.
\end{abstract}

Keywords: Space-time duality, dispersion, nonlinear propagation, optical fiber, Fresnel diffraction.

\section{INTRODUCTION}

The history of conceptions of light is among the most exciting scientific adventures. Debates on the corpuscular or wave nature of light was thus a subject of crucial importance for several centuries, until its dual nature was fully understood. If Young's experiments led to significant progress in understanding the wave behavior of light, it was Fresnel's work that made it possible to establish the most solid theoretical basis of wave optics [1]. The discussions at the French 'Académie des Sciences' held two centuries ago remained particularly famous concerning the experiment that validated Fresnel's approach: according to Fresnel's diffraction theory a counter-intuitive bright spot should appear at the center of the geometric shadow of an illuminated opaque circular object [2]. This phenomenon has remained known as the Arago or Poisson's spot, named after the members of the jury that discussed the results. Since then, Fresnel's wave theory has been widely accepted to describe wave propagation in space and time. Indeed, dispersion and one-dimensional diffraction are linked by a similar mathematical formalism, so that a powerful analogy exists between the temporal and spatial propagation of waves [3-5]. Temporal analogues of common optical systems have been proposed such as lenses [6], diffraction gratings [7], two-waves interferometric devices [8]... This opens up a whole range of new possibilities for ultrafast photonics.

We propose here to further extend the spatial/temporal analogy by revisiting the Arago spot formation in the time domain through the temporal evolution of light after being briefly stopped by an obstacle. We first recall the 1D diffraction/dispersion duality and the modelling of the temporal propagation of light in a linear optical waveguide. In the linear regime of propagation, we show that an analytical treatment confirms that a time domain Arago experiment is relevant. We then present the experiment based on optical fibers and fast optoelectronics. In the final part, we extend our discussion to the impact of Kerr non-linearity.

*christophe.finot@u-bourgogne.fr; phone 33 380395926; fax 33380395971 


\section{ARAGO SPOT FORMATION IN THE LINEAR REGIME}

\subsection{The 1D Arago spot and the space/time duality}

Before discussing our experiments, let us first recall the basis of the analogy between the spatial evolution of light affected by diffraction and the temporal changes experienced by light when dispersion is involved. We consider the simple case where a monochromatic plane wave with wavelength $\lambda$ and an amplitude $a_{0}$ illuminates an opaque strip with a width $2 l$ and an infinite length. In this 1D transverse problem that is illustrated on Fig. 1(b), the longitudinal evolution of light $a(x, z)$ in the scalar approximation is ruled by the following differential equation :

$$
i \frac{\partial a}{\partial z}=-\frac{1}{2 k_{0}} \frac{\partial^{2} a}{\partial x^{2}},
$$

with $x$ and $z$ being the transverse and longitudinal coordinates respectively and $k_{0}=2 \pi / \lambda$ the wavenumber. The two edges of the opaque strip diffract the light leading to the progressive emergence of a bright spot at the center of the wire shadow, as experimentally reported with simple experiments [9-11]. Note that this spot is less intense and spatially broader that in the historic Arago experiment conducted in the space domain and using a 2D opaque perfectly circular screen (Fig. 1(a))

The goal of the present paper is to study the temporal equivalent of a 1D Arago experiment. We therefore consider a fully-coherent continuous wave where light has been switched off for a duration $2 T_{0}$ as illustrated in Fig. 1(c1). This temporal waveform then propagates in a dispersive single mode waveguide (with $\beta_{2}$ being the group velocity dispersion), typically an optical fiber experiencing an evolution of its slowly varying envelope governed in absence of nonlinearity by the following equation [12]:

$$
i \frac{\partial a}{\partial z}=\frac{1}{2} \beta_{2} \frac{\partial^{2} a}{\partial t^{2}}
$$

Dispersion leads to the alteration of the temporal profile (Fig. 1(c2), blue curve): the edges of the waveform have been smoothened, strong oscillations have appeared and a light intensity increase has emerged at the dark pulse center where initially no light was present. This last feature being the temporal counterpart of the Arago spot in this 1D experiment.

The space-time duality readily appears in the mathematical structure of equations (1) and (2) that can be written as:

$$
i \frac{\partial \psi}{\partial \xi}=-\frac{1}{2} \frac{\partial^{2} \psi}{\partial \eta^{2}},
$$

where $\xi$ is a normalized propagation distance $\xi=z / L_{D}$ where $L_{D}$ is defined by $L_{D}=-T_{0}^{2} / \beta_{2}$ (for dispersion, note that with this convention $L_{D}$ is negative for normally dispersive fibers and positive for anomalous dispersion) or $L_{D}=k_{0} l^{2}$ (for diffraction). $\eta$ is the normalized time $\eta=t / T_{0}$ (dispersion) or the normalized transverse coordinate $\eta=x / l$ (diffraction). $\psi=a / a_{0}$ is the field $a$ normalized with respect to the amplitude of the initial plane wave. As a first rough approximation, we can consider that the pattern created by the opaque temporal zone is the result of the superposition of the patterns created by a semi-infinite edges located at $\eta= \pm 1$. Indeed, the field $\psi_{E}$ diffracted by a single initial sharp edge centered at $\eta=0$ is given by:

$$
\psi_{E}(u)=0.5+C(u)+i(0.5+S(u))
$$

where $u=\eta /(\pi \xi)^{1 / 2}$ and $C$ and $S$ are the Fresnel's integrals. This semi-infinite screen (grey dashed line in Fig. 1(c2)) brings some qualitative insight to understand the softening of the edges as well as the nature of the strong fluctuations that develop on each side of the edge in the blue curve. However, the addition of the temporal intensity profiles created by each intensity jump (black line in Fig. 1(c1)) cannot account for the development of the central spot that is the result of a constructive interference process: given the symmetry of our problem, the patterns created by each edge are in phase at the center of the waveform, leading to an increase by a factor 2 of the intensity profile. 

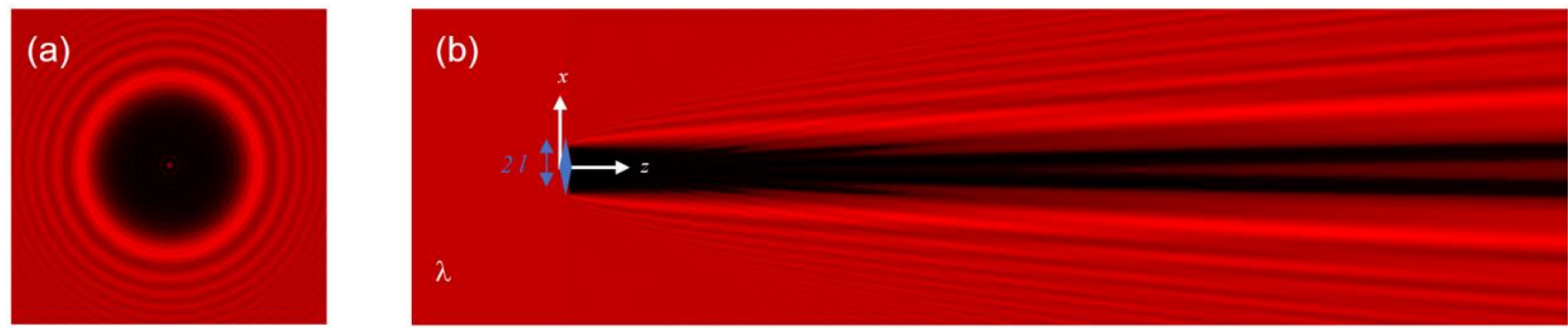

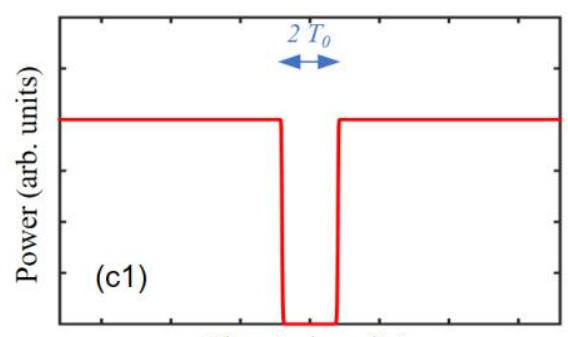

Time (arb. units)

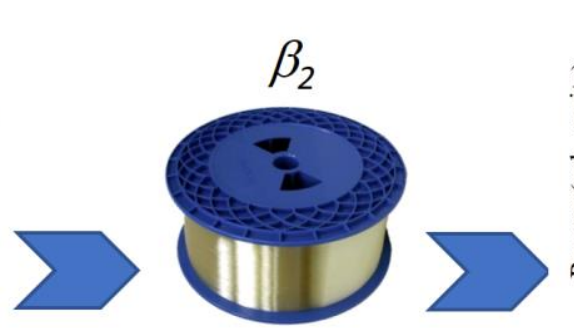

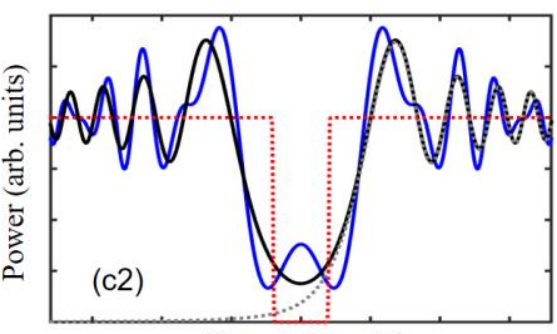

Time (arb. units)

Figure 1. (a) Illustration of the Arago spot resulting from the diffraction of a circular opaque screen when illuminated by a monochromatic wave. (b) Illustration of the longitudinal evolution of the diffraction pattern induced of an opaque strip of width $2 l$. (c) Evolution of the temporal intensity profile of a hole of light. The initial profile (c1) is affected by dispersion. The resulting pattern (c2, blue line) is compared with the shape of diffraction by a semi-infinite screen (grey dotted line) and with the superposition of the intensity pattern resulting from the two edges (solid black line).

\subsection{Analytical results}

The field diffracted by the opaque 1D screen can be fully analytically expressed by means of Fresnel integrals:

$$
\psi_{S}(\eta, \xi)=\frac{1}{\sqrt{2}}\left[\left(1+C\left(\frac{\eta-1}{\sqrt{\pi \xi}}\right)+C\left(\frac{-\eta-1}{\sqrt{\pi \xi}}\right)\right)+i\left(1+S\left(\frac{\eta-1}{\sqrt{\pi \xi}}\right)+S\left(\frac{-\eta-1}{\sqrt{\pi \xi}}\right)\right)\right]
$$

The results are illustrated in the time domain on Fig. 2. Details of the intensity profile for three different propagation distances are provided on panel (a). Whereas the amplitude of the ripple observed on the top level of the wave does not evolve much with propagation distance, the temporal position of the maximum of the oscillations tend to move away from the center of the waveform. Those trends are consistent with the diffraction pattern of a straight edge (dash line in Fig. 2(b1)) and we can note that the maximum value of the oscillation is obtained for $\eta= \pm\left(1+2.12 \xi^{1 / 2}\right)$ with a value of 1.37 expected in this case [13]. We can notice the continuous increase of the central spot with increasing propagation distance, as analytically predicted by :

$$
\left|\psi_{S, 0}(Z)\right|^{2}=2\left[(C(Z)-0.5)^{2}+(S(Z)-0.5)^{2}\right]
$$

with $Z=(\pi \xi)^{-1 / 2}$. The intensity at the center of the waveform therefore continuously increases with the propagation distance $z$ and tends asymptotically to a value equals to the intensity outside of shadow. Equation (6) also confirms analytically that $\left|\psi_{S, 0}(Z)\right|^{2}$ is twice the intensity that may result from the incoherent addition of the intensity linked to each abrupt edge. We complement this study by plotting the temporal phase profile of the diffracted field (panels (b2)). Similarly to the diffraction of a straight edge [13], a significant phase difference exists between the central part and the plateau. This phase difference tends to decrease asymptotically towards 0 . 

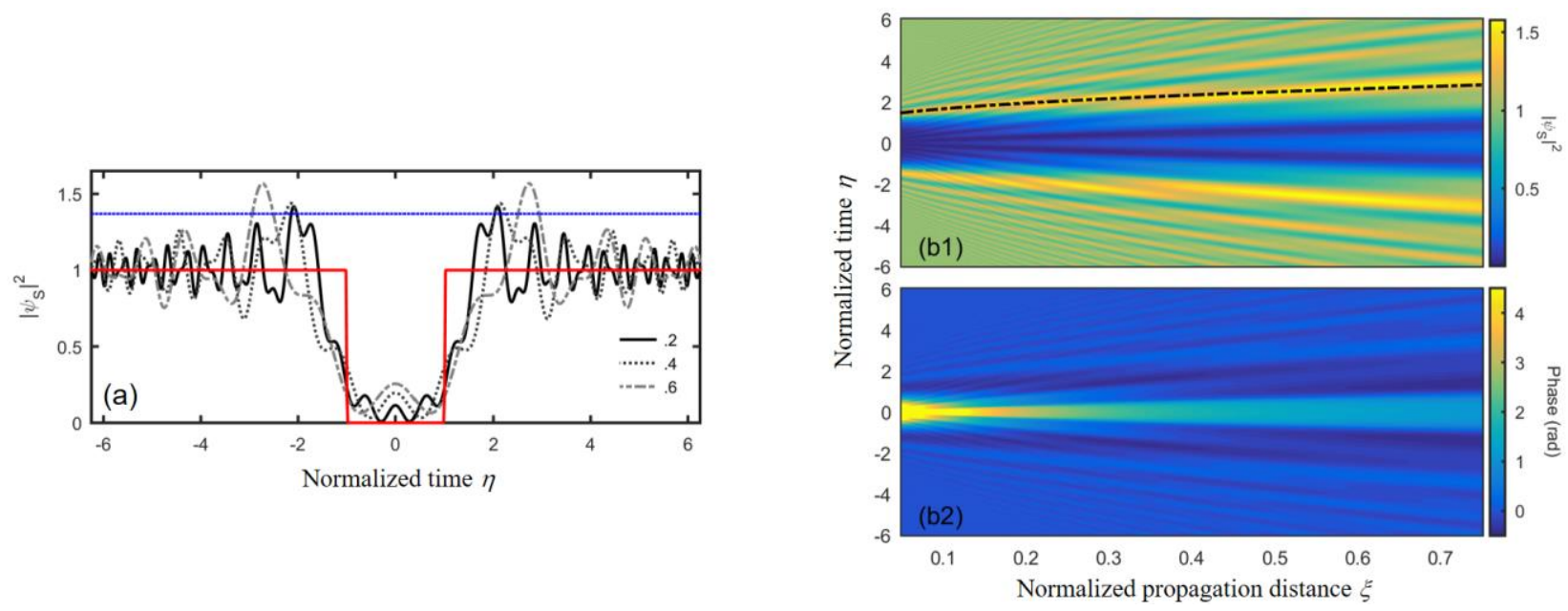

Figure 2. (a) Temporal intensity profiles at three propagation distances $\xi=.2, .4$ and .6. Results are compared with the initial intensity profile (red curve). (b) Longitudinal evolution of the temporal intensity and phase profiles (b1 and b2, respectively). The black dashed line is the analytical prediction of the first maximum of the pattern for a single edge diffraction.

\subsection{Experimental setup}

The experimental setup is sketched in Fig. 3 and relies on devices that are commercially available and used in the telecommunication industry. A laser at $1550 \mathrm{~nm}$ emits a highly-coherent continuous wave. The initial temporal profile is obtained thanks to a Lithium Niobate intensity modulator operated at its point of maximum transmission and driven by an electrical pulse generator that delivers super-Gaussian pulses at a repetition rate of $2.5 \mathrm{GHz}$. The corresponding optical intensity profile is plotted on Fig. 3(b1) (blue solid line) and can be well fitted by an inverted second-order super-Gaussian pulse with a full-width at half duration of $40 \mathrm{ps}$, which is reasonably close from the ideal step edges (red solid line). The initial optical spectrum recorded on a high resolution optical spectrum analyzer confirms the high level of symmetry of the pulse which is close to the Fourier limit and the high degree of coherence of the signal.

(a)
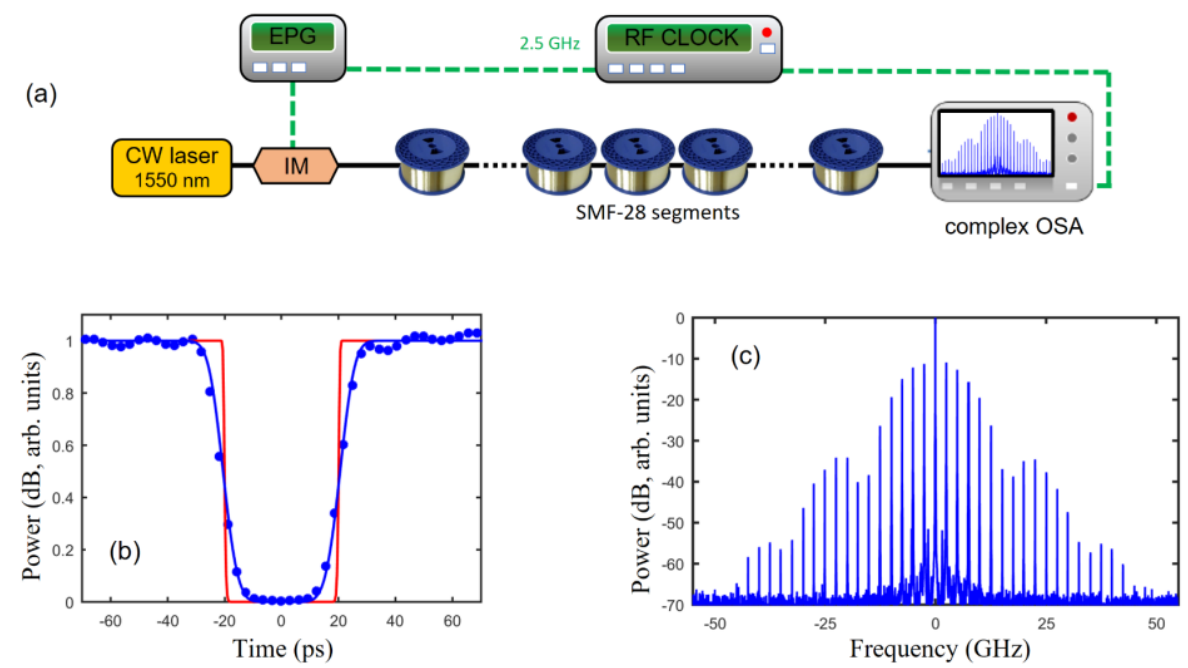

Figure 3. (a) Experimental setup. CW : continuous wave, IM : intensity modulator, EPG : Electrical Pattern Generator, , OSA : Optical Spectrum Analyzer. (b) Temporal intensity profile obtained after modulation. The experimental results recorded with the complex spectrum analyzer (blue circles) are compared with a fit with a second-order super-Gaussian waveform (blue solid line). The red line is the ideal inverted rectangular function. (c) Optical spectrum of the initial signal. 
The linear propagation experiment takes place in a set of optical single mode fibers with anomalous dispersion, i.e. smf28 fibers with a dispersion $\beta_{2}=-20 \mathrm{ps}^{2} / \mathrm{km}$. Using various spools with length between 0.5 and $5 \mathrm{~km}$, we were able to record the longitudinal evolution of the field from $2.5 \mathrm{~km}$ of propagation up to $15 \mathrm{~km}$. The detection of the output pulse properties is ensured by a complex optical spectrum analyzer that enables us to get access both to the spectral intensity and phase profiles as well as their temporal counterparts.

\subsection{Experimental results}

Details of three intensity profiles are provided on Fig. 4 for three propagation distances (4, 8 and $12 \mathrm{~km})$. They are qualitatively in line with the analytical predictions reported in Fig. 2(a): the dispersive propagation is marked by the emergence of oscillations on each edge and a central Arago spot progressively grows.

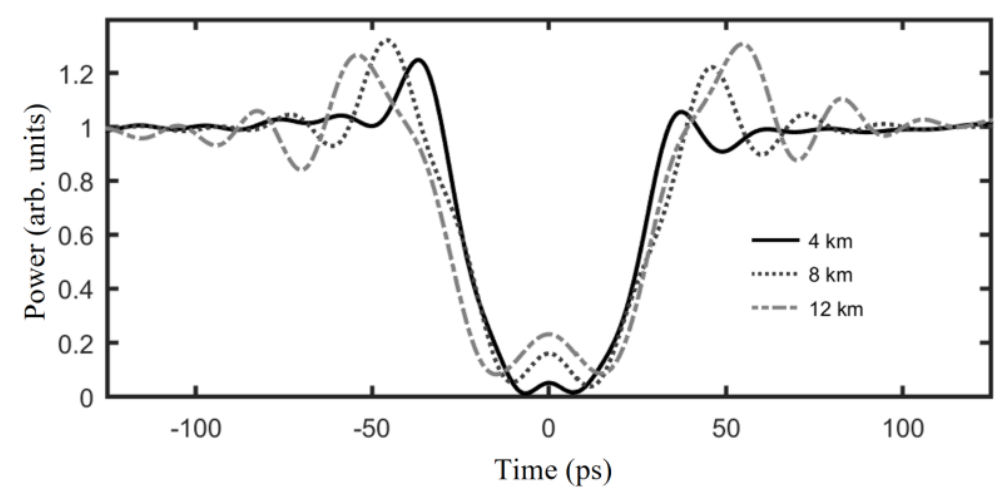

Figure 4. Experimental temporal intensity profiles at three propagation distances $(4,6$ and $8 \mathrm{~km})$ corresponding to $\xi=.2, .4$ and .6 (solid black lines, doted grey lines and mixed grey lines respectively).
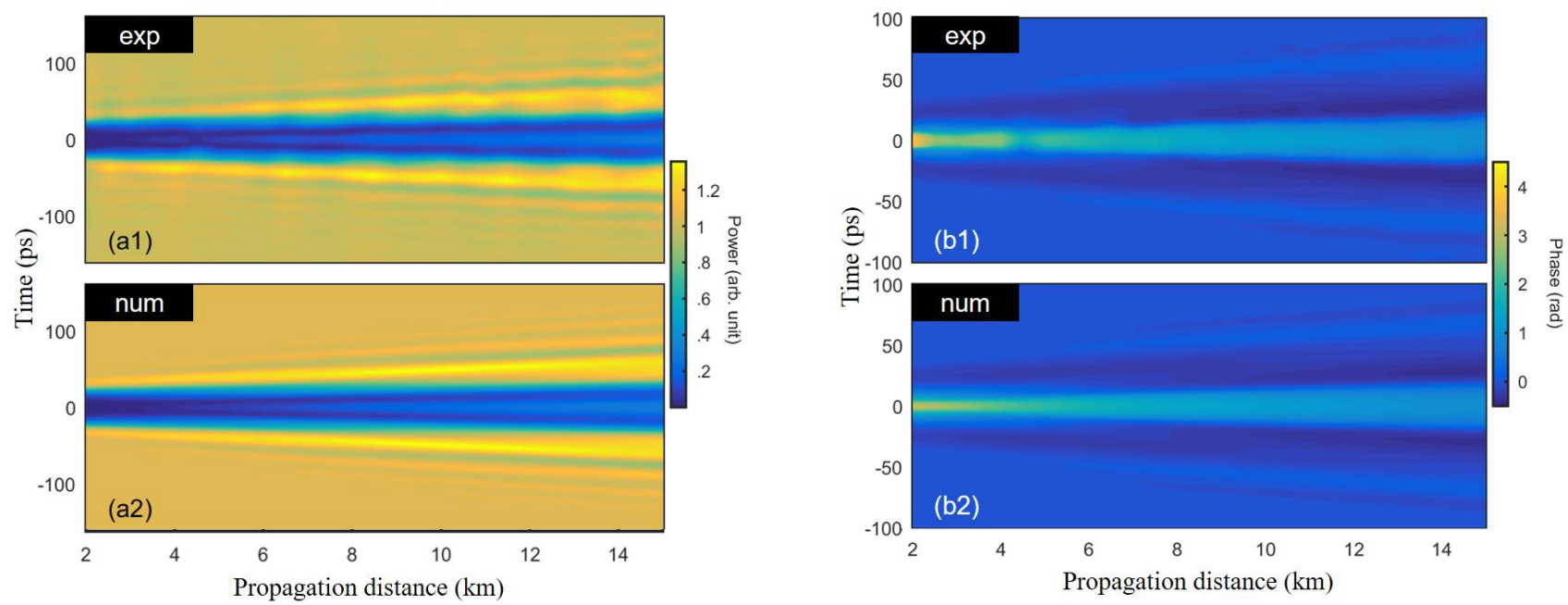

Figure 5. (a) Longitudinal evolution of temporal intensity and phase profiles (panels a and b, respectively). Experimental results (panels 1) are compared with simulations taking into account a second-order super-Gaussian pulse as an initial condition (panels 2). 
A more systematic study made every $500 \mathrm{~m}$ and presented in Fig. 5(a1-b1) enables us to reconstruct the experimental longitudinal evolution of the temporal intensity and phase profiles that are found in qualitative agreement with the theoretical predictions presented in Fig. 2(b). As propagation distance increases, the oscillations move away from the central part but their amplitude does not evolve significantly. On the contrary, the amplitude of the Arago central spot continuously increases. Some deviations can however be noticed for the initial stage of propagation where ideal conditions lead to the emergence of numerous oscillations is the central part. When taking into account the exact initial temporal intensity profiles and when using numerical simulations to solve Eq. (2) (panels 2 of Fig. 5), the agreement regarding the temporal patterns obtained experimentally and numerically becomes quantitative.

\section{ARAGO SPOT FORMATION IN THE NONLINEAR REGIME}

\subsection{Principle}

Contrary to the usual diffraction in free space, propagation in a waveguide can also involve nonlinear effects. Indeed, the temporal evolution of a waveform in an optical fiber is affected by Kerr nonlinearity that can be taken into account through an additional term accounting for self-phase modulation in Eq. (2), leading to the well-known nonlinear Schrödinger equation (NLSE) [12]:

$$
i \frac{\partial a}{\partial z}=\frac{1}{2} \beta_{2} \frac{\partial^{2} a}{\partial t^{2}}-\gamma|a|^{2} a,
$$

with $\gamma$ being the nonlinear coefficient of the fiber. This equation is solved using numerical simulations based on the splitstep Fourier method. The resulting nonlinear dynamics is, as we will see, highly impacted by the regime of dispersion so that studies considering both the normal and anomalous dispersions are required.

From the experimental point of view (see Fig. 6), we have inserted an erbium doped fiber amplifier and two types of fibers have been compared: (1) a SMF-28 fiber (identical to the previously used fiber, with a nonlinear coefficient $\gamma=1.1$ $\mathrm{W}^{-1} \cdot \mathrm{km}^{-1}$ ) with a length of $10 \mathrm{~km}$ and (2) a dispersion shifted fiber with a normal dispersion of $-19 \mathrm{ps}^{2} / \mathrm{km}$, a nonlinear coefficient of $2 \mathrm{~W}^{-1} \cdot \mathrm{km}^{-1}$ and a length of $10.5 \mathrm{~km}$. The two fibers therefore exhibit a rather similar level of integrated dispersion $\left(\approx 200 \mathrm{ps}^{2}\right.$ in absolute value). In order to prevent the deleterious consequences of Brillouin backscattering, we have inserted a phase modulator. Given the additional phase modulation used to prevent Brillouin scattering, it was not possible to take advantage of the complex spectrum analyzer. Therefore, in order to record the temporal intensity profiles, we used a photodiode with a high bandwidth $(70 \mathrm{GHz})$ connected to a high-speed sampling oscilloscope with an electrical bandwidth exceeding $50 \mathrm{GHz}$. Other Brillouin mitigation schemes such as the use of a set of discrete optical isolators could alleviate this strong restriction [14].

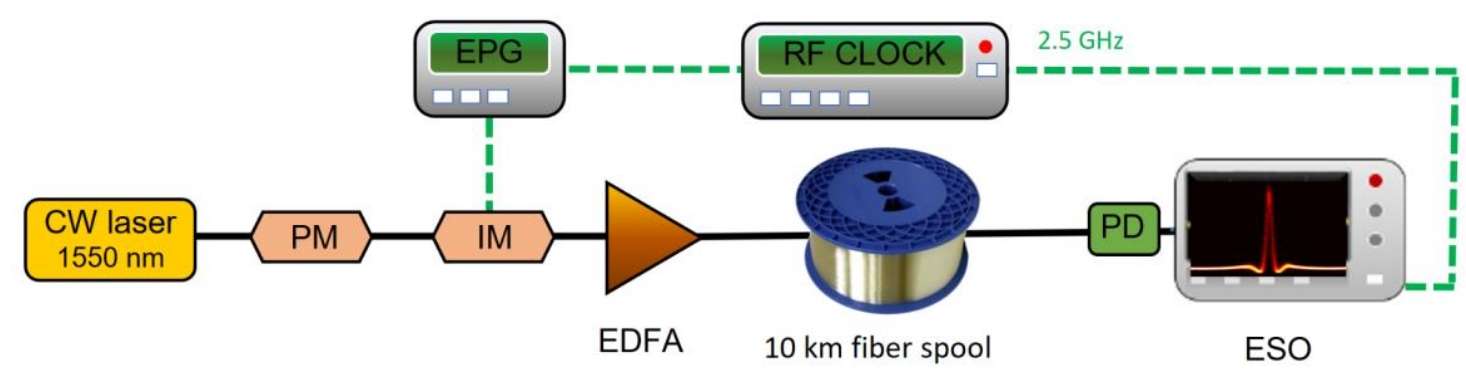

Figure 6. Experimental setup. CW : continuous wave, PM : phase modulator, IM : intensity modulator, EPG : Electrical Pattern Generator, EDFA : Erbium Doped Fiber Amplifier, PD : Photodiode, ESO : Electrical Sampling Oscilloscope. 


\subsection{Results}

The output intensity profiles obtained after propagation in 10-km long fibers with normal or anomalous dispersion are plotted for three levels of input power in Fig. 7(a). For the focusing nonlinearity case (Kerr nonlinearity with anomalous dispersion, panel a1), an increasing input power leads to the progressive development of the lateral oscillations. Such changes in the upper part of the signal have been the subject of recent discussions and can be interpreted in terms of solitons over finite background such as Akhmediev or Peregrine breathers [15]. We also observe a decrease of the central Arago spot when increasing the input power. The opposite behavior is observed for normally dispersive fibers: the central spot is increased whereas the lateral oscillations are lowered (see panel (a2)). The central Arago spot intensity tends to become larger with increasing power. Ultimately, for higher propagation distances or power, the gap tends to be filled and dark solitonic structures may emerge as it has been shown in the study of undular bores [14, 16]. A more systematic study of the output pattern according to the input power is reported on Fig. 7(b) and confirms those trends, showing that the evolution of the Arago central spot is monotonic with power. The experimental results (panel b1) are in excellent agreement with the results provided by the numerical integration of NLSE (panel b2).
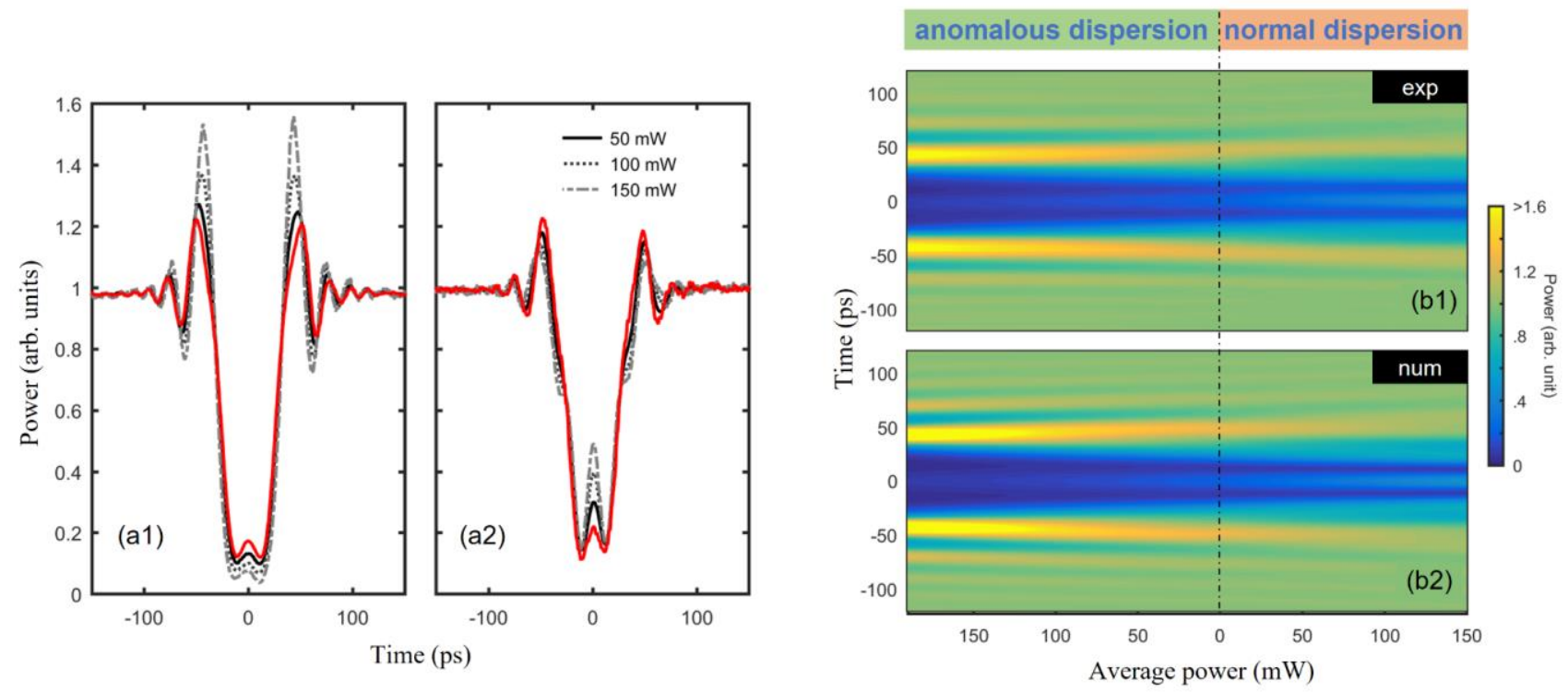

Figure 7. (a) Experimental temporal intensity profiles at different input powers at a fixed propagation distance. The result of linear propagation (red curves) are compared with the temporal profile obtained for input average powers of 50, 100 and 150 $\mathrm{mW}$ (solid black lines, dotted grey lines and dashed grey lines respectively). Measurements performed in the anomalous (a1) and normal (a2) regime of dispersions. (b) Evolution of the temporal intensity profile according to the initial input average power and according to the regime of dispersion. Experimental results (b1) are compared with numerical simulations (b2).

\section{CONCLUSION}

In conclusion, we have investigated theoretically, numerically and experimentally the emergence of a bright spot in the initial shadow of a fully-coherent temporal signal [17]. The studied case is the 1D temporal counterpart of the Arago spot formation that was firstly demonstrated by Fresnel to support his wave theory and acclaimed by Arago in 1817. We have showed that the spot progressively grows with the propagation distance whereas a phase difference exists between the spot center and the surrounding light. As the power increases, the Arago spot intensity is affected by the sign of the dispersion. We finally assess experimentally the existence of the Arago spot formation in the time domain using optical fibers and fast optoelectronics. The longitudinal evolution of a temporal hole of light having sharp edges is studied in a standard smf-28 fiber. The resulting temporal intensity and phase profiles are found in excellent agreement with the analytical or numerical predictions. The experimental results also confirm the influence of the Kerr nonlinearity: a focusing nonlinearity (Kerr effect and anomalous dispersion) tends to decrease the intensity of the spot with increasing power. Moreover, the amplitude of the oscillations that emerge on each side of the shadow increases and the oscillations become more and more abrupt. Opposite trends are observed in a normally dispersive medium where the nonlinearity increases the intensity of the spot. 


\section{ACKNOWLEDGEMENT}

C.F. acknowledges the support by the Région Bourgogne Franche-Comté and the Institut Universitaire de France. H. R. acknowledges the support from the CNRS and Aix-Marseille University.

\section{REFERENCES}

[1] [CEuvres complètes d'Augustin Fresnel] Imprimerie impériale, Paris(1866-1870).

[2] J. E. Harvey, and J. L. Forgham, "The spot of Arago: new relevance for an old phenomenon,” American Journal of Physics, 52(3), 243-247 (1984).

[3] V. Torres-Company, J. Lancis, and P. Andrés, [Chapter 1 - Space-Time Analogies in Optics] Elsevier, 1 (2011).

[4] B. H. Kolner, "Space-time duality and the theory of temporal imaging," IEEE J. Quantum Electron., 30(8), 1951-1963 (1994).

[5] R. Salem, M. A. Foster, and A. L. Gaeta, “Application of space-time duality to ultrahigh-speed optical signal processing," Adv. Opt. Photon., 5(3), 274-317 (2013).

[6] A. A. Godil, B. A. Auld, and D. M. Bloom, "Picosecond time-lenses," IEEE J. Quantum Electron., 30(3), 827837 (1994).

[7] C. Finot, and H. Rigneault, "Experimental observation of temporal dispersion gratings in fiber optics," J. Opt. Soc. Am. B, 34(7), 1511-1517 (2017).

[8] F. Chaussard, H. Rigneault, and C. Finot, "Two-wave interferences space-time duality: Young slits, Fresnel biprism and Billet bilens," Opt. Commun., 397, 31-38 (2017).

[9] A. K. Moen, and D. L. Vander Meulen, "Fresnel Diffraction Using a He-Ne Gas Laser,” Am. J. Phys., 38(9), 1095-1097 (1970).

[10] C. C. Chu, J. D. Hey, and M. Hoarau, “An Optical Diffraction System for Industrial Application,” Phys. Scr., T97(1), 131 (2001).

[11] F. A. Jenkins, and H. E. White, [Fundamentals of optics - 4th edition] McGraw-Hill Inc.,US, (1976).

[12] G. P. Agrawal, [Nonlinear Fiber Optics, Fourth Edition] Academic Press, San Francisco, CA(2006).

[13] M. F. Wolfers, "Diffraction par un bord rectiligne avec une source large," J. Phys. Radium, 6(10), 305-312 (1925).

[14] C. Finot, "Optical pulse doublet resulting from the nonlinear splitting of a super-Gaussian pulse," Laser Phys. Lett., 17, 025103 (2020).

[15] F. Audo, B. Kibler, J. Fatome et al., "Experimental observation of the emergence of Peregrine-like events in focusing dam break flows," Opt. Lett., 43(12), 2864-2867 (2018).

[16] J. Fatome, C. Finot, A. Armaroli et al., "Observation of modulationally unstable multi-wave mixing," Opt. Lett., 38(2), 181-183 (2013).

[17] C. Finot, and H. Rigneault, “Arago spot formation in the time domain,” J. Opt, 21, 105504 (2019). 